\title{
Globular Cluster Metallicity Subpopulations in NGC 4472
}

\author{
Jay Strader ${ }^{1}$, Michael A. Beasley ${ }^{2}$, Jean P. Brodie ${ }^{1}$ \\ strader@ucolick.org, beasley@iac.es, brodie@ucolick.org
}

\begin{abstract}
Bimodality is a common feature of globular cluster (GC) color distributions in galaxies. Although it is well known that the GC system of the Milky Way is bimodal in metallicity, this has yet to be directly demonstrated for an elliptical galaxy. We use Lick index measurements from the literature to derive metallicities for 47 GCs in the giant Virgo elliptical galaxy NGC 4472. The resulting distribution shows clear evidence for two metallicity subpopulations of GCs.
\end{abstract}

Subject headings: globular clusters: general — galaxies: star clusters — galaxies: formation

\section{Introduction}

The color distribution of globular clusters (GCs) in most galaxies is bimodal, with clearly defined subpopulations of blue and red GCs. The peaks of the color distributions correlate with parent galaxy luminosity, and are located at around $V-I \sim 0.95$ and $V-I \sim 1.18$ for massive galaxies (Forbes, Brodie, \& Grillmair 1997; Larsen et al. 2001; Kundu \& Whitmore 2001; Lotz et al. 2004; Strader, Brodie, \& Forbes 2004; Peng et al. 2006; Strader et al. 2006). There is now considerable evidence that GCs in massive galaxies are mostly old (Puzia et al. 2005; Strader et al. 2005) and therefore the color bimodality should be due to metallicity. Typical peak metallicities are then inferred to be $[\mathrm{m} / \mathrm{H}] \sim-1.2$ and $\sim-0.2$ for massive ellipticals. The existence of GC subpopulations leads to important and extensive constraints on models of galaxy formation and evolution (Brodie \& Strader 2006).

Recently, Yoon, Yi \& Lee (2006) have suggested, based on their single stellar population models, that GC color bimodality might instead be due to a nonlinear relation between optical color and metallicity for old (13 Gyr) GCs. The distinguishing aspect of their models

\footnotetext{
${ }^{1} \mathrm{UCO} /$ Lick Observatory, University of California, Santa Cruz, CA 95064

${ }^{2}$ Need this.
} 
(compared to others that show no such effect) is that horizontal branch morphology changes with metallicity in such a way that few GCs have intermediate colors. In the Galaxy, we know that there are indeed two real GC metallicity subpopulations: one associated with the halo and one with the bulge (or possibly thick disk; Zinn 1985). Since there are no obvious differences between the old GC color distributions of spirals and ellipticals, this suggests that genuine metallicity bimodality may be a general phenomenon. Nonetheless, given the wideranging implications of GC subpopulations, it is important to explore the Yoon et al. (2006) idea in detail.

Their hypothesis may be tested in several ways, including deriving metallicity estimates using NIR bands that are unaffected by horizontal branch morphology (e.g., Kundu \& Zepf 2007). Here we employ measurements of Lick indices from the literature for 47 GCs in the massive Virgo elliptical NGC 4472 (Cohen, Blakeslee, \& Côté 2003; hereafter C03). We use two metallicity indicators calibrated on Galactic GCs to determine metallicities for the NGC 4472 GCs, and show that the distribution is indeed bimodal.

\section{Data and Analysis}

C03 presented Lick indices for 47 GCs in NGC 4772 as a subset of a larger study of GC radial velocities in the galaxy (Côté et al. 2003). The GCs studied are among the brightest in the galaxy and have median $\mathrm{S} / \mathrm{N} \sim 20-30$ per resolution element over the $5000-6000 \AA$ range, more than adequate for accurate metallicity estimates. Note that C03 find their GCs to be old ( $\gtrsim 10 \mathrm{Gyr}$ ), so the age-metallicity degeneracy is not an issue in analyzing their spectroscopic indices (see additional discussion in $§ 3.1$ ).

At the time of the observations, Keck/LRIS did not yet have an optimized blue arm, so the spectral coverage of the data was restricted to the red, and only a subset of the Lick indices could be measured. Here we study six indices: $\mathrm{H} \beta, \mathrm{Mg}_{1}, \mathrm{Mg}_{2}, \mathrm{Mg} b, \mathrm{Fe} 5270$, and Fe5335. Four redder indices were also measured ( $\mathrm{NaD}, \mathrm{TiO} 1, \mathrm{TiO} 2$, and $\mathrm{H} \alpha)$, but since these are difficult to measure accurately for Galactic GCs (because of interstellar extinction and stochastic measurement effects), we omit them from subsequent analysis.

It is important to note that C03 did not measure indices using the modern Lick definitions (e.g., Trager et al. 1998) but rather using the older definitions in Burstein et al. (1984). The differences are small shifts in the passband locations because of an updated wavelength calibration.

We estimated the effect of the different definitions using our calibration data set of 39 Galactic GCs from Schiavon et al. (2004). We removed two GCs from the original set of 
41: NGC 6544 and NGC 7078. The former has indices that lie off the tight relations of the rest of the GCs; the latter GC is much more metal-poor than any of the rest of the sample $([\mathrm{m} / \mathrm{H}] \sim-2.3)$, and the correlation between index strength and metallicity becomes quite nonlinear at very low metallicities. The remaining GCs cover a range of metallicity from $[\mathrm{m} / \mathrm{H}] \sim-1.8$ to solar and this encompasses most of the system. These metallicities were taken from the catalog of Harris (1996) and are not on a well-defined scale, which is why we use the "agnostic" term $[\mathrm{m} / \mathrm{H}]$ instead of $[\mathrm{Fe} / \mathrm{H}]$ or $[Z / \mathrm{H}]$ (see Strader \& Brodie 2004 for additional discussion). Note that the age-metallicity relationship of the Galactic GC data is built into our calibrations.

We measured the six indices under study using both the modern Lick definitions and using the Burstein et al. (1984) definitions, the latter after smoothing the spectra to the $\sim 6$ $\AA$ resolution of the $\mathrm{C} 03$ data. The resulting offsets are quite small for most of the atomic indices (ranging from $\sim 0$ to $0.2 \AA$; this confirms a result from $\mathrm{C} 03$ ), but are relatively large for the two molecular indices $\left(\mathrm{Mg}_{1}\right.$ and $\left.\mathrm{Mg}_{2}\right)$, amounting to $\sim 0.02 \mathrm{mag}$. This corresponds to a change of $0.2-0.3 \mathrm{dex}$, depending on metallicity. Fortunately, the offsets have little or no metallicity dependence, so we corrected the NGC 4472 data for these offsets and proceeded to use calibrations calculated with the standard index definitions. Additional small offsets could be present due to differences in the flux calibration between the Galactic and NGC 4472 data, but previous works have found such changes to be small, even for relatively wide molecular indices (e.g., Larsen \& Brodie 2002).

Yoon et al. (2006) noted that the Mgb indices of GCs in M87 (Cohen, Blakeslee, \& Ryzhov 1998) were not bimodal, as might be expected if the GC metallicity distribution had two subpopulations. In retrospect this is perhaps not surprising, since there is a colorluminosity relation for blue GCs in M87 (Strader et al. 2006), and the color distribution for bright GCs (those sampled by Cohen et al. 1998) is close to unimodal as the blue peak merges into the red one. Curiously, unlike many other giant ellipticals, NGC 4472 shows no evidence for this "blue tilt" of metal-poor GCs (Strader et al. 2006; Harris et al. 2006; Mieske et al. 2006). Figure 1 shows that the distribution of Mgb indices for NGC 4472 GCs is clearly bimodal.

\subsection{Metallicity Estimates}

We estimate metallicities for the NGC 4472 GCs using two methods. The first is based on a principal components analysis (PCA) of the Galactic GC indices. Strader \& Brodie (2004) performed a PCA on 11 Lick indices using the Schiavon et al. (2004) data; we refer the reader to the former paper for additional details. The main conclusion from Strader \& 
Brodie (2004) is that the first principal component (PC1) of the Lick indices of Galactic GCs is metallicity, and that a linear combination of normalized Lick indices provides an accurate estimate of $[\mathrm{m} / \mathrm{H}]$ for GCs (with an rms scatter of only $0.12 \mathrm{dex}$ ).

Here we repeat their analysis using the six indices discussed above. The results are nearly identical: $95 \%$ of the variance in the indices is in PC1. As in Strader \& Brodie (2004), each of the indices has nearly equal weighting. PC1 correlates strongly with $[\mathrm{m} / \mathrm{H}]$, and we fit a second-order polynomial between $\mathrm{PC} 1$ and $[\mathrm{m} / \mathrm{H}]$ to capture the slight nonlinearity in the points. The residual standard error is 0.12 dex.

While reasonably accurate, PCA metallicities have a flaw-there is no good way to estimate errors. We also chose to use a method well-developed in the literature: using each index separately to measure the metallicity and then using the dispersion in these values as an estimate of the error. While calibrations exist (e.g., Brodie \& Huchra 1990), none use the set of indices available in the NGC 4472 data.

We fit second-order polynomials between the Galactic GC indices and $[\mathrm{m} / \mathrm{H}]$. Each fit is reasonable, with residual standard errors of $0.13-0.2$ dex depending on the index. Since the PCA indicates that each index contributes approximately the same amount of information to $[\mathrm{m} / \mathrm{H}]$, there is no need to weight some indices more heavily than others. To determine a $[\mathrm{m} / \mathrm{H}]$ and error from the resulting estimates, we use robust indicators: a Tukey biweight for the mean $[\mathrm{m} / \mathrm{H}]$ and the median absolute deviation for the error. The latter quantity is the median value of the absolute difference between the individual $[\mathrm{m} / \mathrm{H}]$ values and the biweight mean, and is then scaled to be equivalent to $\sigma$ for an asymptotically normal distribution. We term the resulting $[\mathrm{m} / \mathrm{H}]$ values "composite" metallicities and denote them with $[\mathrm{m} / \mathrm{H}]_{c}$.

Figure 2 plots the literature $[\mathrm{m} / \mathrm{H}]$ estimates vs. $[\mathrm{m} / \mathrm{H}]_{P C A}$ and $[\mathrm{m} / \mathrm{H}]_{c}$ for Galactic GCs. The two estimates are quite consistent over the metallicity range $-1.8 \lesssim[\mathrm{m} / \mathrm{H}] \lesssim 0$. We proceed below using $[\mathrm{m} / \mathrm{H}]_{c}$ so that we can assign errors to our metallicity estimates.

\section{Results}

Composite metallicities were determined for the NGC 4472 GCs using the calibration from $\S 2.1$, after the index definition correction described in $\S 2$. These $[\mathrm{m} / \mathrm{H}]_{c}$ values are given in Table 1 and a histogram is plotted in Figure 3. Despite the small number of objects, bimodality is clear in the plot: there are two subpopulations, with mean metallicities of $[\mathrm{m} / \mathrm{H}]_{c} \sim-1.1$ and 0 . A kernel density estimate with a bin width of 0.1 dex is overplotted and is consistent with the visual impression of bimodality. We have also plotted a similar density estimate for Galactic GCs from the Harris (1996) catalog. The two peaks in the Galactic 
GC distribution are shifted to lower metallicities than in NGC 4472; this is a consequence of the GC metallicity-galaxy luminosity relations that exist for both subpopulations (Larsen et al. 2001; Strader et al. 2004; Lotz et al. 2004; Strader et al. 2006; Peng et al. 2006). Figure 3 suggests that the peak metallicity differences between the subpopulations in the Milky Way and NGC 4472 are $\sim 0.4$ dex and $\sim 0.5$ dex for the metal-poor and metal-rich GCs, respectively. These offsets are slightly larger than the typical values predicted by the published relations (see, e.g., Brodie \& Strader 2006), but are within the intrinsic scatter of the relations.

We tested statistically for bimodality in the NGC 4472 metallicities using KMM (Ashman et al. 1994) and Nmix (Richardson \& Green 1997; see discussion in Strader et al. 2006). Both fit mixture models of normal distributions to data. For KMM, the $p$-value was 0 for both heteroscedastic and homoscedastic fits, strongly indicating two Gaussians are a better fit to the data than one. For the heteroscedastic fit, the resulting distributions had peaks of $[\mathrm{m} / \mathrm{H}]_{c}=-1.10$ and -0.01 and $\sigma=0.41$ and 0.13 . NMix gave very similar results: $p<10^{-5}$ that the data are unimodal, and best-fit distributions with parameters of $[\mathrm{m} / \mathrm{H}]_{c}=-1.14$ and -0.03 with $\sigma=0.38$ and 0.18 . These values are close to the approximate peak locations estimated in C03.

Our results are consistent with those of C03. They derived metallicities using four indices (Mgb, Fe5270, Fe5335, and NaD) and stellar population models. We use no models but are inextricably tied to Galactic GCs. While they did not explicitly demonstrate metallicity bimodality, Figure 4 shows that our estimates fall close to a one-to-one relation with theirs; at the highest metallicities, C03 find more GCs with very supersolar values. Our errors are somewhat lower, probably due to our use of a larger number of indices.

\subsection{Potential Issues}

There are three potential issues affecting our conclusions: the accuracy of the metallicity scale, the effect of age (or hot star) variations, and whether this analysis can be extrapolated to the entire GC system of NGC 4472. Here we show that none of these has a significant impact on our conclusion that there are two GC metallicity subpopulations in NGC 4472.

The metallicity scale of Galactic GCs in Harris (1996) is not well-defined; there are GC $[\mathrm{m} / \mathrm{H}]$ values on both the Zinn \& West (1984) and Carretta \& Gratton (1997) scales and ill-defined combinations of the two (indeed, this is probably the cause of much of the scatter

\footnotetext{
${ }^{1}$ Using the C03 metallicities on the Zinn \& West (1984) scale.
} 
in the calibrations of $[\mathrm{m} / \mathrm{H}])$. There are other concerns. Our sample of Galactic GCs has few metal-rich objects. The $[\alpha / \mathrm{Fe}]$ ratio of NGC $4472 \mathrm{GCs}$ could be different than in the Galaxy, though C03 find no strong evidence for this. We could have systematically over- or underestimated the mean $[\mathrm{m} / \mathrm{H}]_{c}$ of the metal-rich NGC 4472 GCs by $\sim 0.15-0.25$ dex. However, since we find the mean $[\mathrm{m} / \mathrm{H}]_{c}$ to be close to solar, it seems unlikely that we have underestimated by a large amount. We make no claims about the reality of NGC 4472 GCs with very supersolar metallicities, but given that we find a separation of $\sim 1$ dex in the metallicities of the two subpopulations, the errors cannot account for the clear bimodality.

The second issue is whether there are age differences in the GC sample; through the agemetallicity degeneracy such variations could affect the estimated metallicities. Non-canonical hot star populations could have a similar effect. C03 find that the spread in $\mathrm{H} \beta$ indices is consistent with observational errors at all metallicities, so there is no evidence for either a GC age spread or strong horizontal branch variations at fixed metallicity. However, since there is probably a slight age-metallicity relation among Galactic GCs (with the metal-rich GCs younger by $\sim 1-2 \mathrm{Gyr}$ ), this relation is built into our metallicity calibration. This could induce a systematic error if the NGC 4472 GCs had a different age-metallicity relation than the Galaxy. Fortunately, the effect is small. For example, if the NGC 4472 metal-rich GCs had ages of 13 Gyr (instead of $~ 11$ Gyr as in the Galaxy; Salaris \& Weiss 2002), we would only have overestimated their metallicities by $\sim 0.05$ dex. It is also important to note that if the metal-rich GCs were $\lesssim 10 \mathrm{Gyr}$, the Yoon et al. (2006) models would not predict a nonlinear color-metallicity relation. And while a blue horizontal branch in a metal-rich GC might mimic a young age in the Balmer lines, the effect on the metal lines is much smaller, and the only Balmer line in our calibration is $\mathrm{H} \beta$. Maraston (2005) models that include a rather extreme blue horizontal branch in metal-rich single stellar populations show an effect of only $\sim 5-20 \%$, depending on the specific metal line and the assumed metallicity of the population. Thus we conclude that GC age or hot star effects are not the cause of the metallicity bimodality.

The final question is whether the sample of NGC 4472 GCs from C03 is representative of the system as a whole. These GCs nearly all have $T_{1}<21.5$ and so are among the brightest GCs in the galaxy, and the $C-T_{1}$ color distribution of this sample is very close to that of the GC system of NGC 4472 as a whole (see Figure 16 in C03). This suggests that metallicity bimodality is a common feature of the entire GC system. In Figure 5 we show $C-T_{1}$ vs. $[\mathrm{m} / \mathrm{H}]_{c}$ for the NGC 4472 GCs. Also plotted are color-metallicity relations, derived from Galactic GCs, from Geisler \& Forte (1990) and Harris \& Harris (2002). The two are very similar except at high metallicities, where the Harris \& Harris (2002) relation swings sharply upward. Given the uncertainty in the metallicity scale, both provide adequate fits over the $[\mathrm{m} / \mathrm{H}]$ range covered by the NGC 4472 data. 
Even if our GC sample is representative in terms of colors, is it plausible that its relatively small size is leading to a spurious detection of bimodality? Yoon et al. (2006) argued that, in their models, the color distribution of M87 GCs can be produced by a single normal distribution in metallicity, with $[\mathrm{m} / \mathrm{H}] \sim-0.6$ and $\sigma \sim 0.5$. We tested this by drawing random samples of $47 \mathrm{GCs}$ from this distribution and then analyzing the results as for the

original NGC 4472 GCs. Out of 10000 draws, $\sim 4 \%$ favor bimodality over unimodality. The majority of these have a central unimodal population with a few objects at a widely separated metallicity, quite unlike the NGC 4472 distribution. Less than $1 \%$ of the total number of simulations have bimodality similar to that seen in NGC 4472, even when we allow for peak separations smaller than that observed. This analysis shows that it is unlikely that the metallicity bimodality in NGC 4472 GCs is a statistical fluke.

\section{Discussion}

Most recent papers in the literature have assumed the existence of GC metallicity bimodality, so we do not feel the need to explore the implications of our findings. However, we should stress what we have not shown. The exact form of the optical color-metallicity relation for GCs is still poorly determined, and it will be challenging to improve it as long as the $[\mathrm{m} / \mathrm{H}]$ scale for Galactic GCs, especially at the metal-rich end, is ill-defined. There may still be significant nonlinearities of the sort Yoon et al. (2006) propose. We have shown that - at least in the case of NGC 4472 - these are not the primary cause of the color bimodality. To directly test their color-metallicity relation, a galaxy with a large population of very old ( $\sim 13 \mathrm{Gyr})$, intermediate-metallicity GCs is required.

An important implication of the Yoon et al. (2006) work is that the detailed age distribution of the GC system may be important in determining GC colors. Horizontal branch morphologies can change substantially with age changes of only 1-2 Gyr. Thus, it is possible that the color-metallicity relationship may not be universal, even at old ages. The number of elliptical galaxies with large, high-S/N samples of GC spectra is still distressingly small. Given that GCs will continue to be used as calibrators for stellar population models, the need for more data in this area is urgent.

We acknowledge support by the National Science Foundation through Grant AST0507729 . 


\section{REFERENCES}

Ashman, K. M., Bird, C. M., \& Zepf, S. E. 1994, AJ, 108, 2348

Brodie, J. P., \& Huchra, J. P. 1990, ApJ, 362, 503

Brodie, J. P., \& Strader, J. 2006, ARA\&A, 44, 193

Burstein, D., Faber, S. M., Gaskell, C. M., \& Krumm, N. 1984, ApJ, 287, 586

Carretta, E. \& Gratton, R. G. 1997, A\&AS, 121, 95

Cohen, J. G., Blakeslee, J. P., \& Côté, P. 2003, ApJ, 592, 866

Cohen, J. G., Blakeslee, J. P., \& Ryzhov, A. 1998, ApJ, 496, 808

Côté, P., McLaughlin, D. E., Cohen, J. G., \& Blakeslee, J. P. 2003, ApJ, 591, 850

Forbes, D. A., Brodie, J. P., \& Grillmair, C. J. 1997, AJ, 113, 1652

Geisler, D., \& Forte, J. C. 1990, ApJ, 350, L5

Harris, W. E. 1996, AJ, 112, 1487

Harris, W. E., \& Harris, G. L. H. 2002, AJ, 123, 3108

Harris, W. E., Whitmore, B. C., Karakla, D., Okoń, W., Baum, W. A., Hanes, D. A., \& Kavelaars, J. J. 2006, ApJ, 636, 90

Kundu, A. \& Whitmore, B. C. 2001, AJ, 121, 2950

Kundu, A. \& Zepf, S. E. 2007, ApJ, submitted

Larsen, S. S., Brodie, J. P., Huchra, J. P., Forbes, D. A., \& Grillmair, C. J. 2001, AJ, 121, 2974

Larsen, S. S. \& Brodie, J. P. 2002, AJ, 123, 1488

Lotz, J. M., Miller, B. W., \& Ferguson, H. C. 2004, ApJ, 613, 262

Maraston, C. 2005, MNRAS, 362, 799

Mieske, S., et al. 2006, ApJ, 653, 193

Peng, E. W., et al. 2006, ApJ, 639, 95

Puzia, T. H., et al. 2005, A\&A, 439, 997 
Richardson, S. \& Green, P. G. 1997, JR Statist. Soc. B, 1997, 59, 731

Salaris, M., \& Weiss, A. 2002, A\&A, 388, 492

Schiavon, R. P., Rose, J. A., Courteau, S., \& MacArthur, L. A. 2004, ApJ, 608, L33

Strader, J., \& Brodie, J. P. 2004, AJ, 128, 1671

Strader, J., Brodie, J. P., \& Forbes, D. A. 2004, AJ, 127, 295

Strader, J., Brodie, J. P., Cenarro, A. J., Beasley, M. A., \& Forbes, D. A. 2005, AJ, 130, 1315

Strader, J., Brodie, J. P., Spitler, L., \& Beasley, M. A. 2006, AJ, 132, 2333

Trager, S. C., Worthey, G., Faber, S. M., Burstein, D., \& Gonzalez, J. J. 1998, ApJS, 116, 1

Yoon, S.-J., Yi, S. K., \& Lee, Y.-W. 2006, Science, 311, 1129

Zinn, R. \& West, M. J. 1984, ApJS, 55, 45

Zinn, R. 1985, ApJ, 293, 424 


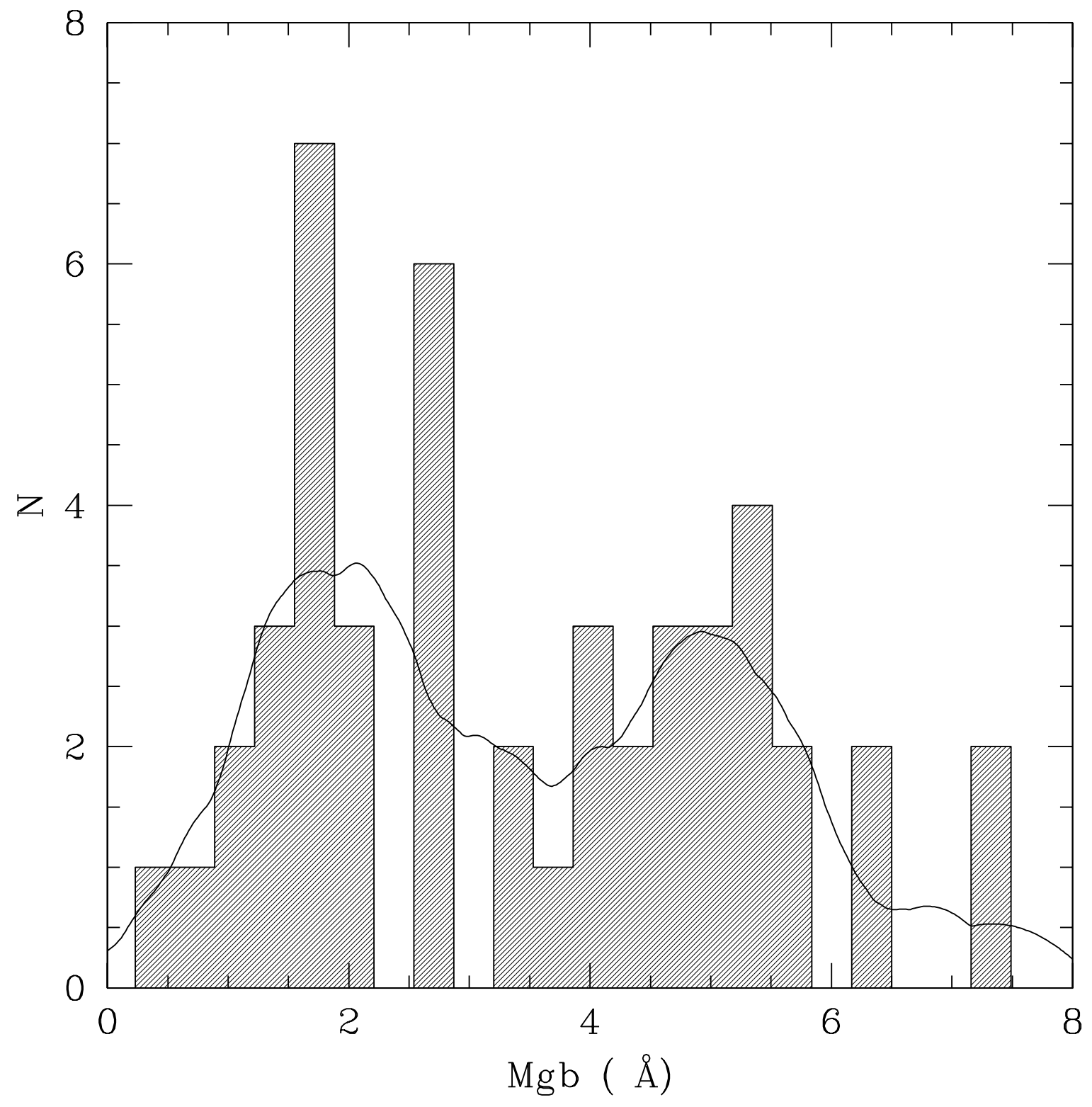

Fig. 1.- Histogram of Mgb index strength for NGC 4472 GCs. A density estimate with an Epanechnikov kernel (bin width $0.4 \AA$ ) is overplotted. 


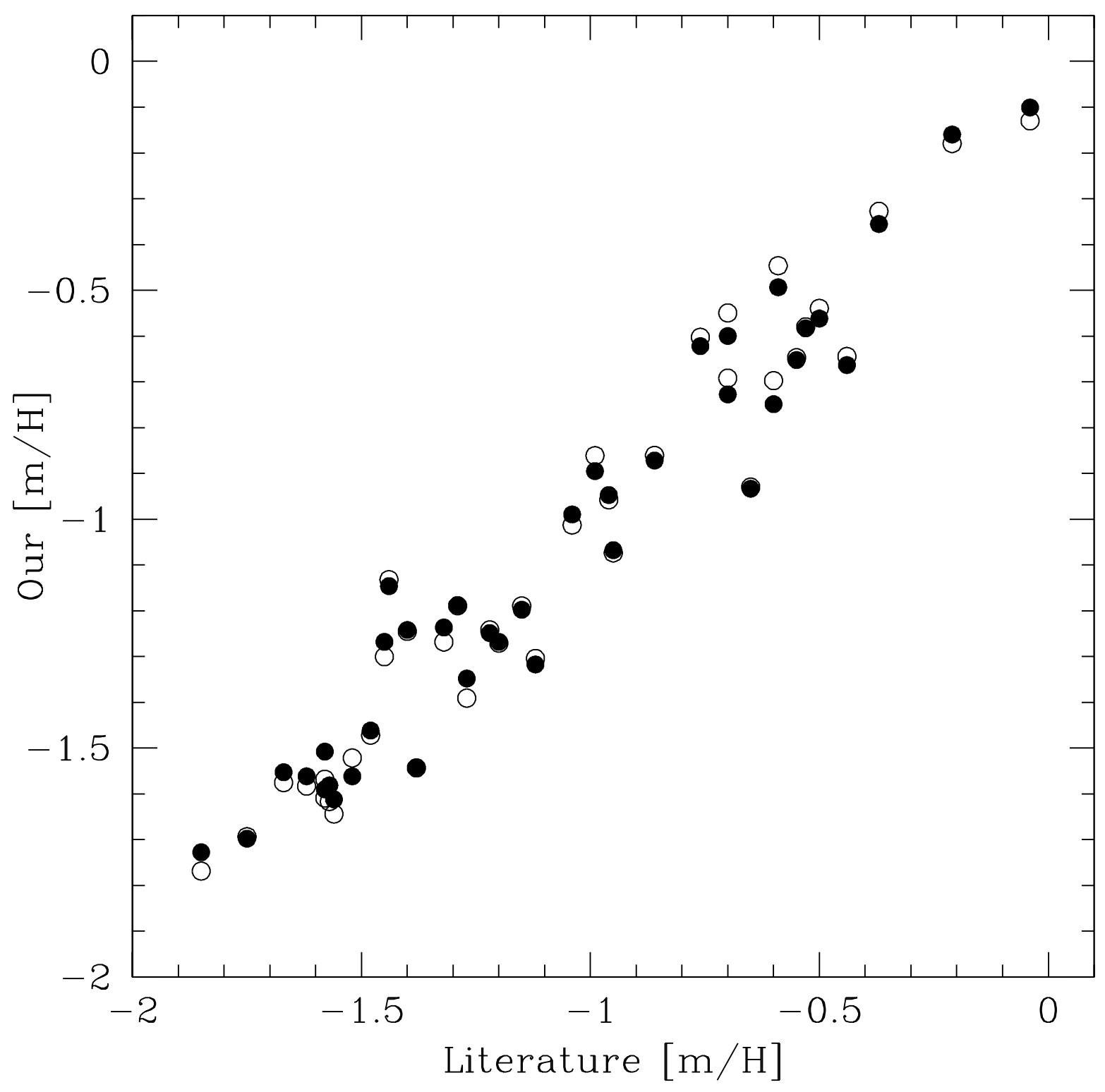

Fig. 2.- Literature $[\mathrm{m} / \mathrm{H}]$ for Galactic GCs vs. $[\mathrm{m} / \mathrm{H}]_{P C A}$ (filled circles) and $[\mathrm{m} / \mathrm{H}]_{c}$ (open circles) derived from our calibrations. 


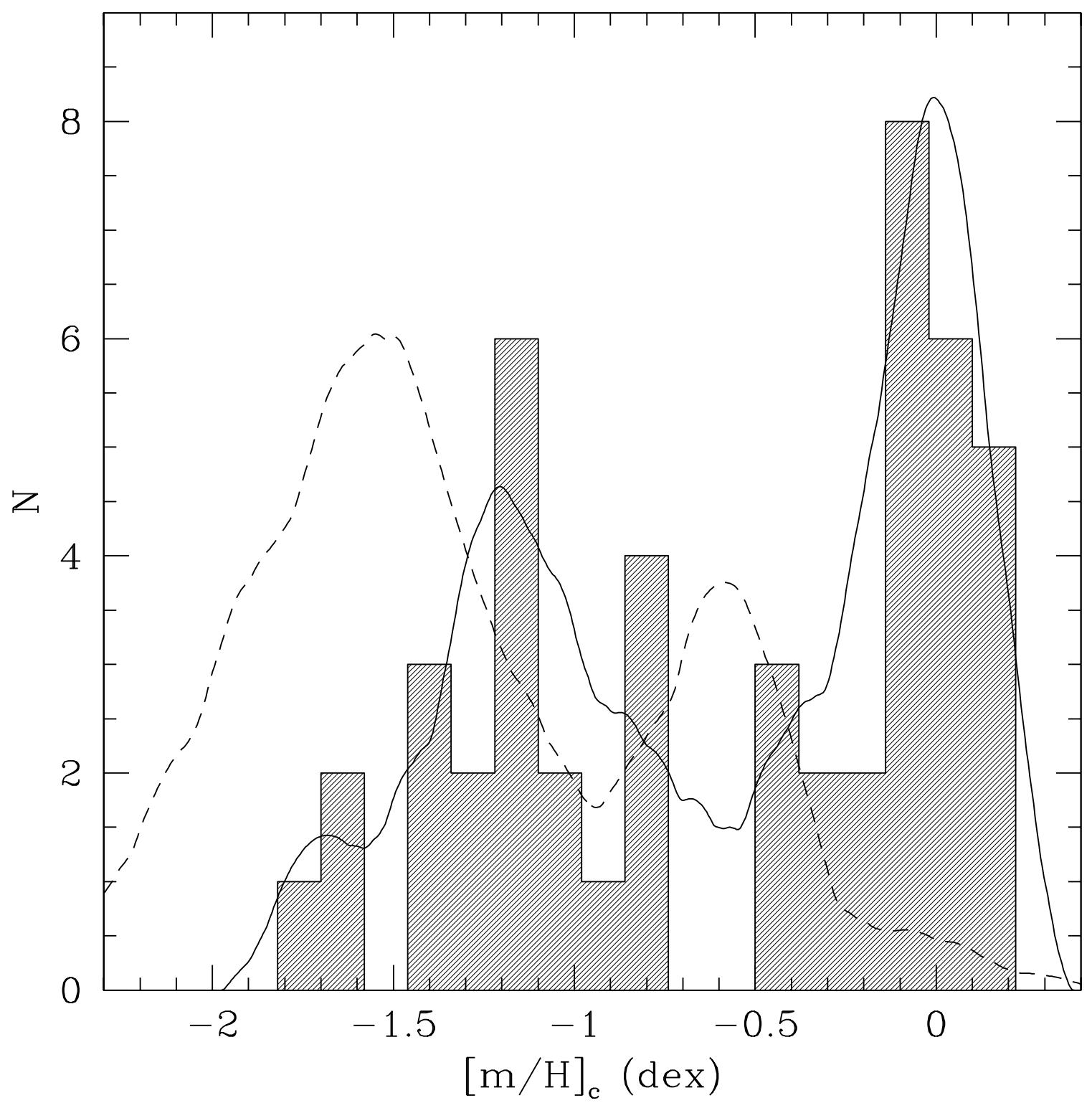

Fig. 3.- Histogram of $[\mathrm{m} / \mathrm{H}]_{c}$ for NGC 4472 GCs. Density estimates with an Epanechnikov kernel (bin width 0.1 dex) for NGC 4472 (solid line) and the Milky Way (dashed lines) are overplotted. The difference in peak locations is a direct result of the mean GC metallicitygalaxy luminosity relationships for both subpopulations. 


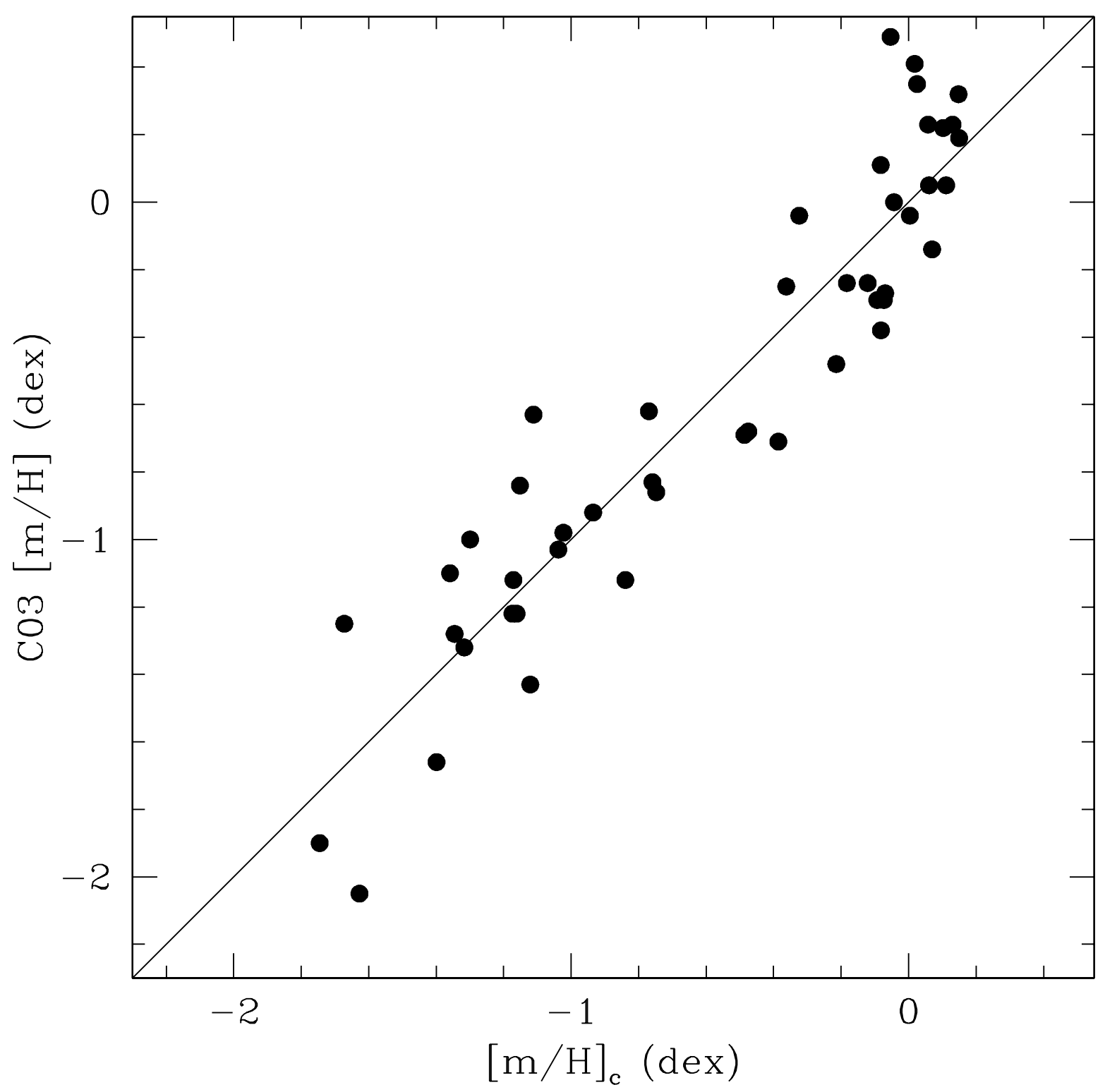

Fig. 4.- A comparison of metallicity estimates for NGC 4472 GCs between C03 and this paper. The solid line is a one-to-one relation; in the mean the two estimates are quite consistent. 


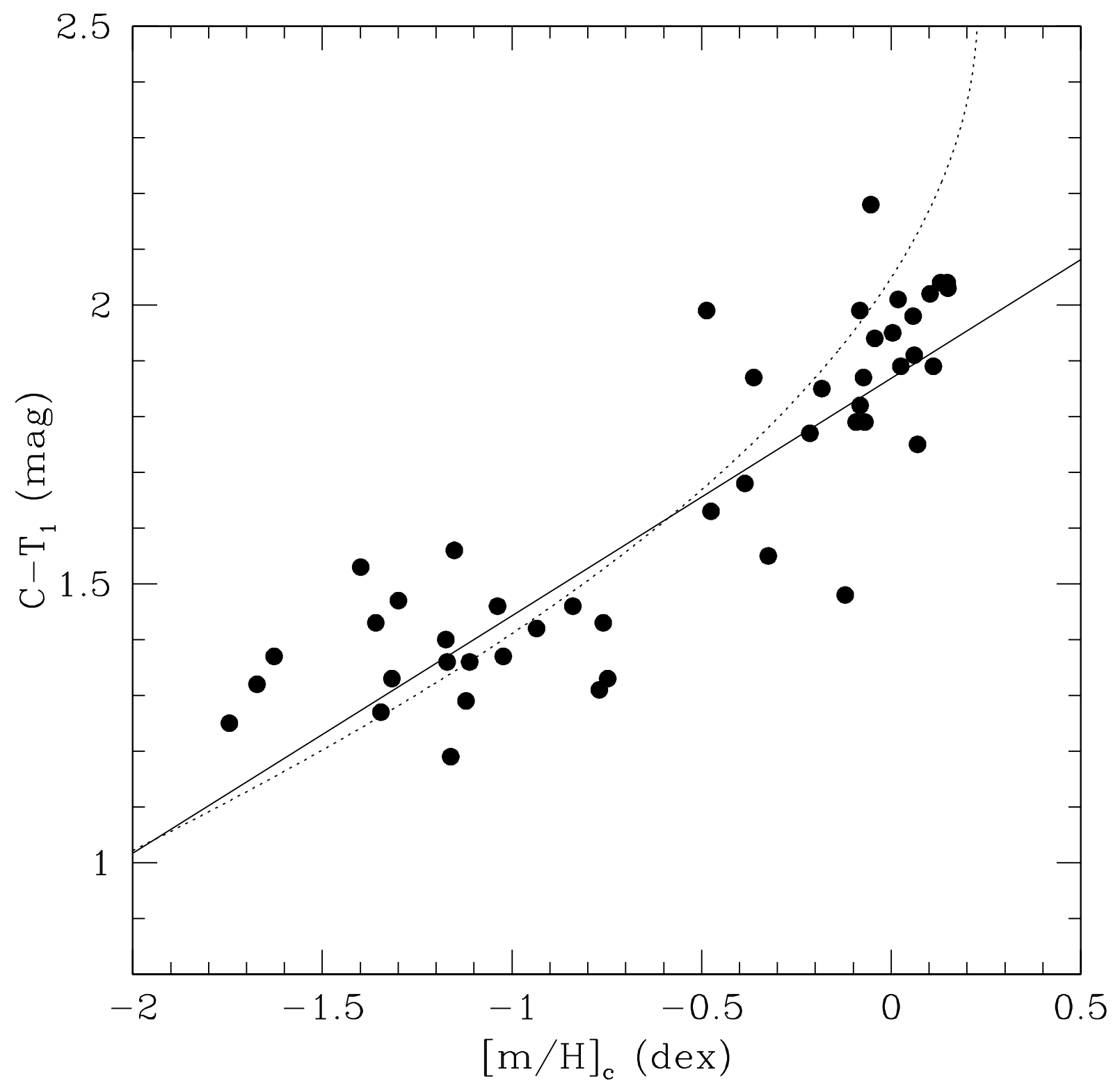

Fig. 5. $-C-T_{1}$ vs. $[\mathrm{m} / \mathrm{H}]_{c}$ for NGC 4472 GCs. The overplotted lines are color-metallicity relations for Galactic GCs from Geisler \& Forte (1990; solid) and Harris \& Harris (2002; dashed). 
Table 1. Data for NGC 4472 Globular Clusters ${ }^{\mathrm{a}}$

\begin{tabular}{|c|c|c|c|c|}
\hline ID & $\begin{array}{c}T_{1} \\
(\mathrm{mag})\end{array}$ & $\begin{array}{c}C-T_{1} \\
(\mathrm{mag})\end{array}$ & $\begin{array}{r}{[\mathrm{m} / \mathrm{H}]_{c}} \\
(\mathrm{dex})\end{array}$ & $\begin{array}{c}{[\mathrm{m} / \mathrm{H}]_{c} \text { error }} \\
(\mathrm{dex})\end{array}$ \\
\hline 1475 & 21.15 & 1.46 & -0.84 & 0.15 \\
\hline 1508 & 21.49 & 1.99 & -0.08 & 0.09 \\
\hline 1650 & 20.85 & 1.95 & 0.00 & 0.12 \\
\hline 1731 & 20.71 & 1.82 & -0.08 & 0.08 \\
\hline 1798 & 20.69 & 1.98 & 0.06 & 0.11 \\
\hline 1846 & 21.07 & 2.02 & 0.10 & 0.06 \\
\hline 1889 & 20.98 & 1.25 & -1.75 & 0.14 \\
\hline 1892 & 21.09 & 1.53 & -1.40 & 0.53 \\
\hline 1905 & 21.22 & 1.36 & -1.11 & 0.12 \\
\hline 2013 & 21.28 & 1.40 & -1.17 & 0.17 \\
\hline 2031 & 20.71 & 1.37 & -1.02 & 0.03 \\
\hline 2045 & 20.94 & 1.77 & -0.21 & 0.17 \\
\hline 2060 & 20.62 & 1.29 & -1.12 & 0.19 \\
\hline 2178 & 21.51 & 1.19 & -1.16 & 0.24 \\
\hline 2188 & 21.15 & 1.33 & -0.75 & 0.13 \\
\hline 2306 & 20.35 & 1.63 & -0.48 & 0.22 \\
\hline 2406 & 20.85 & 2.03 & 0.15 & 0.08 \\
\hline 2421 & 21.09 & 1.43 & -1.36 & 0.08 \\
\hline 2502 & 21.08 & 1.48 & -0.12 & 0.03 \\
\hline 2528 & 20.34 & 1.46 & -1.04 & 0.11 \\
\hline 2543 & 20.27 & 1.36 & -1.17 & 0.05 \\
\hline 2569 & 20.12 & 1.89 & 0.11 & 0.02 \\
\hline 2813 & 21.00 & 1.94 & -0.04 & 0.12 \\
\hline 3150 & 21.40 & 1.79 & -0.09 & 0.06 \\
\hline 3603 & 20.47 & 1.75 & 0.07 & 0.06 \\
\hline 3788 & 20.80 & 1.87 & -0.36 & 0.42 \\
\hline 3900 & 21.04 & 1.89 & 0.03 & 0.13 \\
\hline 4017 & 20.92 & 1.42 & -0.93 & 0.16 \\
\hline 4062 & 20.77 & 2.01 & 0.02 & 0.01 \\
\hline 4144 & 20.74 & 1.33 & -1.32 & 0.14 \\
\hline
\end{tabular}


Table 1-Continued

\begin{tabular}{lccrc}
\hline \hline ID & $\begin{array}{c}T_{1} \\
(\mathrm{mag})\end{array}$ & $\begin{array}{c}C-T_{1} \\
(\mathrm{mag})\end{array}$ & $\begin{array}{r}{[\mathrm{m} / \mathrm{H}]_{c}} \\
(\mathrm{dex})\end{array}$ & $\begin{array}{c}{[\mathrm{m} / \mathrm{H}]_{c} \text { error }} \\
(\mathrm{dex})\end{array}$ \\
\hline 4168 & 20.36 & 1.68 & -0.39 & 0.08 \\
4217 & 20.66 & 1.79 & -0.07 & 0.14 \\
4296 & 20.79 & 1.27 & -1.35 & 0.18 \\
4351 & 20.38 & 1.37 & -1.63 & 0.25 \\
4401 & 20.85 & 1.91 & 0.06 & 0.01 \\
4513 & 20.10 & 1.85 & -0.18 & 0.17 \\
4541 & 20.83 & 1.56 & -1.15 & 0.07 \\
4663 & 20.38 & 1.87 & -0.07 & 0.02 \\
4682 & 21.42 & 1.55 & -0.32 & 0.13 \\
4834 & 20.24 & 1.47 & -1.30 & 0.13 \\
4852 & 21.13 & 2.04 & 0.13 & 0.09 \\
4864 & 20.63 & 1.99 & -0.49 & 0.15 \\
5003 & 20.72 & 1.43 & -0.76 & 0.14 \\
5018 & 20.70 & 2.04 & 0.15 & 0.06 \\
5097 & 20.76 & 2.18 & -0.05 & 0.18 \\
5217 & 20.60 & 1.32 & -1.67 & 0.22 \\
6051 & 20.94 & 1.31 & -0.77 & 0.06 \\
\hline
\end{tabular}

aThe photometry is taken from Côté et al. (2003) 